\title{
Psicologia da Saúde Crítica no Contexto Hospitalar
}

Critical Health Psychology In The Hospital Context

Psicología De La Salud Crítica En El Contexto Hospitalario

Denis Barros de Carvalho

Universidade

Federal do Piauí

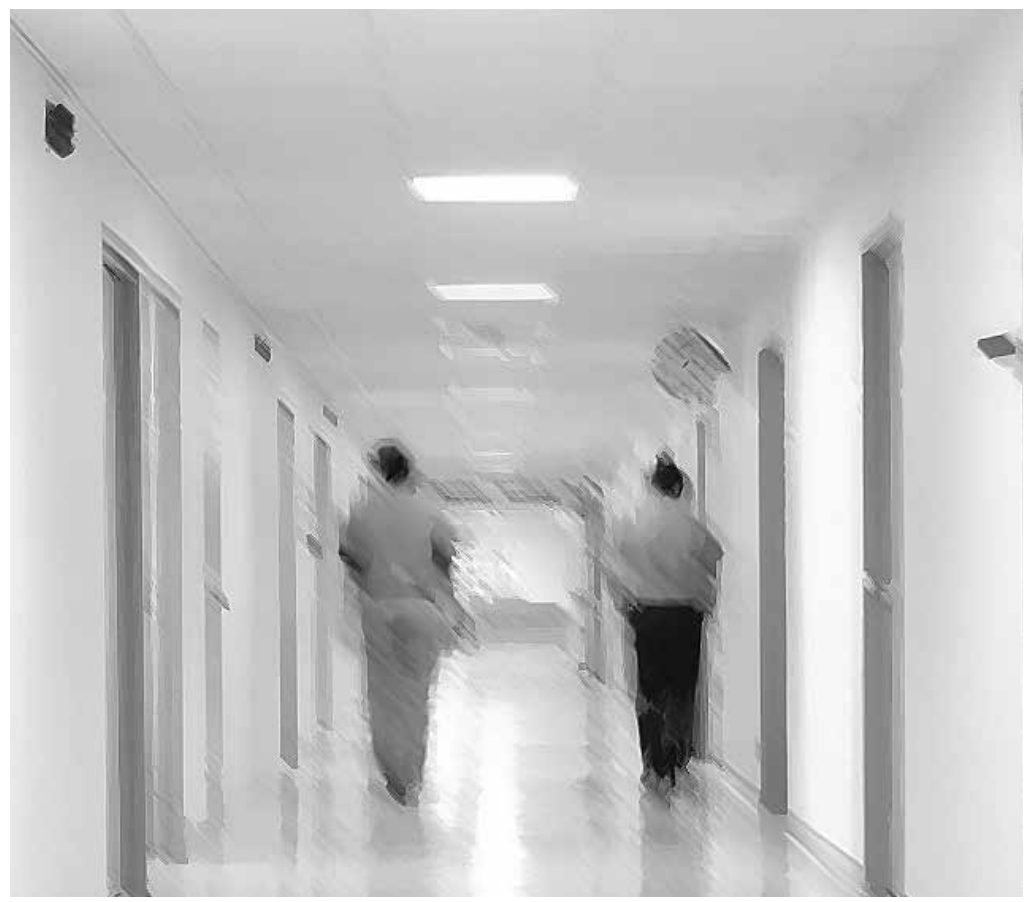


Resumo: O texto apresenta uma discussão sobre a Psicologia da saúde no contexto hospitalar a partir de uma perspectiva histórica e ética, mostrando como o desenvolvimento desse campo foi caracterizado pelo surgimento de duas perspectivas: a Psicologia da saúde tradicional e a Psicologia da saúde crítica. O modelo de quatro abordagens à Psicologia da saúde é apresentado e reformulado com a inclusão do conceito de Psicologia sanitária, e a prática em Psicologia da saúde é discutida à luz do conceito de reflexividade. Por fim, alguns questionamentos derivados da Psicologia da saúde crítica são feitos à prática do psicólogo no contexto hospitalar.

Palavras-chave: Psicologia da saúde. História da Psicologia. Psicologia hospitalar. Atuação do psicológo.

\begin{abstract}
This paper presents a discussion about health psychology in the hospital from a historical and ethical perspective. The essay explains how the development of this field was characterized by the emergence of two perspectives: the traditional health psychology and the critical health psychology. The model of four approaches to health psychology is presented and reformed to include the concept of general health psychology, and the practice in health psychology is discussed from the point of view of the concept of reflexivity. Finally, some questions derived from critical health psychology are asked about the psychologist's practice in the hospital setting.
\end{abstract}

Keywords: Health psychology. History of psychology. Hospital psychology. Psychologist performance.

Resumen: El texto presenta una discusión sobre la Psicología de la salud en el contexto hospitalario desde una perspectiva histórica y ética, mostrando como el desarrollo de ese campo fue caracterizado por el surgimiento de dos perspectivas: la Psicología de la salud tradicional y la Psicología de la salud crítica. El modelo de cuatro abordajes a la Psicología de la salud es presentado y reformulado con la inclusión del concepto de Psicología sanitaria, y la práctica en Psicología de la salud es discutida a partir del concepto de reflexividad. Para finalizar, algunos cuestionamientos derivados de la Psicología de la salud crítica son realizados a la práctica del psicólogo en el contexto hospitalario.

Palabras clave: Psicología de la salud. Historia de la Psicología. Psicología hospitalaria. Actuacion del psicologo.

Os estudos brasileiros sobre a inserção da Psicologia no contexto hospitalar se concentram, predominantemente, nas questões referentes à formação profissional e de configuração de modelos e estratégias de práticas psicológicas (Yamamoto \& Cunha, 1998; Yamamoto, Trindade, \& Oliveira, 2002; Castro \& Bornholdt, 2004; Marcon, Luna, \& Lisboa, 2004; Sá, Lima, Santos, \& Clemente, 2005; Tonetto \& Gomes, 2005; Tonetto \& Gomes, 2007a, 2007b), com certo destaque, no que diz respeito à formação profissional, de trabalhos que descrevem experiências de estágios (Guedes, 2006; Maia, Silva, Martins, \& Sebastiani, 2005).

Tonetto e Gomes, em um estudo empírico sobre a prática dos psicólogos em hospitais de Porto Alegre, afirmam que a Psicologia hospitalar se apresenta como uma nova área de atuação, embora reconheçam que os recursos teórico-metodológicos que são utilizados nesse contexto não diferem tecnicamente dos que aparecem em contextos diferentes. A prática dos psicólogos hospitalares reafirmaria o caráter generalista da formação em Psicologia. Os autores se fundamentam na Resolução no. 14/2000, do Conselho Federal de Psicologia (CFP), que define a Psicologia hospitalar como uma especialidade, para justificarem a constituição do espaço hospitalar como uma nova área de atuação para os psicólogos brasileiros. Tal posição contrasta frontalmente com a perspectiva adotada em dois trabalhos produzidos por Oswaldo Yamamoto e colaboradores.

No primeiro, Yamamoto e Cunha (1998) argumentam que o hospital é um local, e não um campo de atuação, o que torna pouco defensável sua classificação como área de atuação. No segundo, Yamamoto, Trindade e Oliveira descartam a denominação Psicologia hospitalar por duas razões: a primeira, a inadequação do uso de um local de trabalho para designar uma área de atuação, e a segunda é que a identificação por local 
Castro e

Bornholdt (2004)

preferem incluir

a Psicologia

hospitalar na

área ampla

da Psicologia

da saúde,

que utilizaria

conhecimentos

das ciências

biomédicas, da

Psicologia clínica

e da Psicologia

comunitária

para intervir

em diversos

contextos

sanitários,

incluindo o

hospital. tende a pulverizar e a fragmentar o campo profissional da Psicologia, tornando muito difícil a construção de uma identidade de profissional de saúde para o psicólogo que atua no contexto hospitalar.

Castro e Bornholdt (2004) preferem incluir a Psicologia hospitalar na área ampla da Psicologia da saúde, que utilizaria conhecimentos das ciências biomédicas, da Psicologia clínica e da Psicologia comunitária para intervir em diversos contextos sanitários, incluindo o hospital. As autoras foram as primeiras a refletir sobre a especificidade da Psicologia hospitalar no Brasil, tendo como parâmetro a realidade internacional. Em contraste com os modelos estadunidense e espanhol, nos quais o marco conceitual da Psicologia da saúde serve de base para o trabalho do psicólogo no contexto hospitalar, a Psicologia brasileira transformou um local de trabalho em uma área de atuação.

Castro e Bornholdt concordam com Yamamoto e Cunha e com Yamamoto, Trindade e Oliveira (2002) e Chiattone (2000) quando declaram que seria mais adequado referirse ao trabalho do psicólogo no contexto hospitalar como parte da Psicologia da saúde. Apesar da importância do trabalho de Castro e Bornholdt, alguns questionamentos podem ser feitos a esse artigo. Em primeiro lugar, é possível apresentar um quadro internacional mais completo com referências à Psicologia da saúde desenvolvida em outros países, como Cuba (Bernal, Sosa, Alfonso, Gómez, \& García, 2009), Canadá (Arnett, 2006), e, principalmente, Portugal (Teixeira, Cima, \& Santa Cruz, 1999; Teixeira, 2004; Trindade \& Teixeira, 2002).

O modelo cubano é consequência das transformações pelas quais o país passou em função da Revolução de 1959, principalmente com a criação do Sistema Nacional de Saúde, que possibilitou a introdução do psicólogo na atenção primária e modificou a concepção do trabalho psicológico em hospitais, ampliando a atuação para outros contextos além do psiquiátrico. Assim, o psicólogo passou a trabalhar em todos os contextos hospitalares e a lidar com o processo saúde-doença. Em consequência dessa inserção, a formação do psicólogo cubano para área de saúde foi modificada, com a criação de três figuras formativas: a maestría em Psicologia de la Salud (criada em 1993), a especialização em Psicologia de la Salud (criada em 1995) e a licenciatura em Psicologia, ênfase em saúde (criada em 2004). Assim, foi criado em Cuba um Sistema de Formação em Psicologia da Saúde que abrangia o sistema superior de ensino (Bernal et. al., 2009).

A Psicologia da saúde no Canadá, por sua vez, apresenta-se profundamente envolvida com o sistema de saúde pública daquele país (Kenkel, Deleon, Mantell, \& Steep, 2005; Romanov \& Marchildon, 2003; Arnett, 2006; Westra, Eastwood, Bouffard, \& Gerritsen, 2006), sendo marcada pela transição entre um trabalho restrito ao contexto das instituições de saúde mental para um envolvimento com a saúde integral (Kenkel et al., 2005). Além disso, o contexto hospitalar tem se firmado como um espaço privilegiado dos psicólogos da saúde no contexto canadense (Humbke, Brown, Welder, Fillion, Dobson, \& Arnett, 2004).

Em Portugal, a Psicologia da saúde começou a ser organizada a partir da década de 80 , sendo centrada principalmente na questão da doença e nos enfoques derivados da Psicologia clínica (Teixeira, 2002). O desenvolvimento da área pode ser verificado pela organização de uma Sociedade Portuguesa de Psicologia da Saúde, de congressos, revistas e livros. A produção teórica tem discutido o conceito de Psicologia da saúde e analisado suas vertentes teóricas (Teixeira, 2004; Correia, 2006; Teixeira, 2008). O hospital é visto como local de intervenção do psicólogo da saúde, sendo que ela se diferencia em intervenção 
junto aos pacientes, intervenção com técnicos e funcionários e intervenção junto à organização (Trindade \& Teixeira, 2002).

Outra complementação necessária ao artigo de Castro e Bornholdt é a afirmação da pluralidade existente no campo, o que uma leitura apressada das autoras supramencionadas poderia negar. Há, pelo menos, duas perspectivas atualmente no campo da Psicologia da saúde: uma Psicologia da saúde tradicional e uma Psicologia da saúde crítica (Crossley, 2000; Teixeira, 2004). A Psicologia da saúde tradicional possui um modelo biopsicossocial de saúde e, metodologicamente, assenta-se em estratégias quantitativas de investigação. Essa área se centra no indivíduo como âmbito da Psicologia e utiliza um modelo de comportamento de risco e seu valor preditivo para definir os determinantes do processo saúde-doença. A Psicologia da saúde crítica, por sua vez, trabalha com um modelo de produção social da saúde, utiliza preferencialmente estratégias qualitativas de investigação e investiga as experiências de saúde e doença a partir do estudo de suas significações sociais.

O propósito deste trabalho é duplo: apresentar o desenvolvimento histórico da Psicologia da saúde, mostrando o surgimento das perspectivas tradicional e crítica, e também, a partir da Psicologia da saúde crítica, apresentar algumas questões para o trabalho do psicólogo no contexto hospitalar. O texto divide-se em quatro partes: na primeira, é apresentado um histórico da Psicologia da saúde, com um relato e discussão acerca do surgimento das perspectivas tradicional e crítica; na segunda parte, discute-se as quatro abordagens à Psicologia da saúde, a partir do modelo original de David Marks, psicólogo inglês (2002); na terceira, a prática da Psicologia da saúde é discutida a partir da perspectiva crítica, e, na quarta e última parte, algumas questões sobre o trabalho do psicólogo da saúde no contexto hospitalar são apresentadas e discutidas a partir da perspectiva crítica.

\section{Da Psicologia da saúde tradicional à Psicologia da saúde crítica}

A constituição da Psicologia da saúde como um campo autônomo de pesquisa e de intervenção psicológicas ocorre efetivamente, no contexto anglo-saxônico, no final da década de 70. O marco inaugural dessa constituição é a criação da trigésima oitava seção da Associação de Psicologia Americana, cujo primeiro presidente, Joseph Matarazzo (1980), apresentou um comunicado dirigido à Divisão de Psicologia da Saúde na Reunião Anual da Associação de Psicologia Americana (APA), no qual explicitava o modelo teórico que predominaria no campo. Seu texto, intitulado Behavioral Health and Behavioral Medicine - Frontiers for a New Health Psychology (Saúde Comportamental e Medicinal Comportamental - Fronteiras para uma Nova Psicologia) reflete a influência do comportamentalismo como corrente psicológica hegemônica nos Estados Unidos naquela ocasião. Matarazzo apresentou aquela que é considerada a primeira definição de Psicologia da saúde:

Psicologia da saúde é um agregado das específicas contribuições educacionais, científicas e profissionais da disciplina da Psicologia à promoção e manutenção da saúde, à prevenção e ao tratamento das doenças e à identificação dos correlatos etiológicos e diagnósticos da saúde, da doença e das disfunções relacionadas (1980, p.815)

Em um artigo posterior, Matarazzo (1982) acrescentaria os seguintes propósitos ao novo campo teórico-prático: análise e melhoramento do sistema de saúde e formação de políticas de saúde. Uma das questões pouco discutidas sobre o texto inaugural do 
autor é sua perspectiva individualista. Adriane Roso (2007), por exemplo - em um texto sobre Psicologia social da saúde - mostra que vivemos em um contexto histórico dominado por uma cosmovisão individualista-liberal que impossibilitou a emancipação humana e a promoção da saúde para todos. A autora cita o conceito de Psicologia da saúde de Matarazzo (1980, 1982), mas não problematiza seu enfoque individualista.

Matarazzo cita, com aprovação de J. H. Knowles, médico e filósofo social estadunidense:

Muitos indivíduos não se preocupam com a saúde até o momento em que a perdem... Eu acredito que a idéia de um 'direito' à saúde (garantido pelo governo) deve ser substituída pela idéia de uma obrigação moral do indivíduo de preservar sua própria saúde - um dever público, se assim você quiser (Knowles, p.59, como citado em Matarazzo, 1980, p. 809)

O próprio Matarazzo reafirma essa posição a definir da seguinte forma a saúde comportamental:

Saúde comportamental é um campo interdisciplinar dedicado a promover uma filosofia da saúde que enfatiza a responsabilidade individual na aplicação de técnicas das ciências biomédicas e comportamentais na manutenção da saúde e na prevenção de doenças e disfunções através de uma variedade de atividades propostas ou de iniciativa própria do indivíduo (1980, p. 813)

É possível concordar com Marks (2002) quando este afirma que a posição assumida por Matarazzo é ideológica e não científica, tomando a forma de um discurso moral. Marks afirma que é necessário discutir quais são os propósitos de um sistema de saúde pública e que tipo de sistema de saúde os psicólogos e outros trabalhadores desejam defender. É preciso um debate aberto sobre os princípios morais e éticos que nos justifica tentar persuadir as pessoas a mudarem de comportamento. Contudo, é também possível acrescentar outra crítica filosófica: o conceito de cuidado de saúde é baseado em um modelo competitivo-individualista da relação entre indivíduo e sociedade que desconsidera a base social de toda ação humana (Kelman, 1980).

Matarazzo assinala que o desenvolvimento de um modelo biopsicossocial na Medicina teve alguma importância para o campo da saúde. Ele cita o trabalho de George Engel (1977), sem considerá-lo de fato inovador. Santiago-Delfosse e Chamberlain (2008), todavia, consideram o texto The Need for a New Medical Model: a Challenge for Biomedicine, de Engel, como iniciador da Psicologia da saúde. Psiquiatra interessado na Medicina psicossomática, Engel (1977) não se dirige especificamente aos psicólogos, mas procura contribuir para evitar uma cisão na psiquiatria de sua época, que se encontrava em meio a um intenso debate entre uma corrente biológica e uma psicodinâmica, esta última derivada da psicanálise. O autor propõe uma concepção de psiquiatria mais aberta às correntes psicodinâmicas e também uma pluralidade metodológica, acolhendo estratégias quantitativas e qualitativas de investigação (Santiago-Delfosse \& Chamberlain, 2008). Apesar disso, o artigo de Engel era primariamente uma crítica ao modelo biomédico, e somente na sua segunda metade aparecem algumas referências ao que seria um modelo biopsicossocial, o que é demonstrado por meio de exemplos, e não pelo desenvolvimento de uma teoria, de um modelo ou de uma abordagem (Stam, 2000). Marks (2002) afirma que Engel nunca definiu o que seria o modelo psicossocial, deixando essa definição em aberto e possibilitando que diferentes concepções fossem elaboradas.

Por fim, Matarazzo (1980, 1982) apresenta uma justificativa econômica para o desenvolvimento da Psicologia da saúde: o gasto de $10 \%$ do Produto Interno Bruto 
estadunidense poderia ser evitado se houvesse melhor desenvolvimento do comportamento individual para a compreensão da saúde. O estilo de vida individual relacionado a hábitos negativos seria responsável pelo declínio da saúde de muitos americanos (Matarazzo, 1980). A Psicologia aparece, então, como a ciência mais bem qualificada para os desafios da mudança do comportamento (Matarazzo, 1984). Essa compreensão individualista de situações complexas, aliada a uma visão economicista, somente é compreensível a partir do entendimento do seu contexto: o debate sobre os custos da assistência médica, privada e pública, ocorrido nas décadas de 70 e 80 nos Estados Unidos (Santiago-Delfosse \& Chamberlain, 2008). De acordo com Robert Crawford (2006), o sucesso do projeto de privatização e de entrega às leis do mercado dos problemas sociais somente foi possível com o triunfo do discurso da responsabilidade individual sobre a saúde em detrimento da responsabilidade coletiva, o que teria ocorrido entre 1975 e 1985. Em outras palavras, de acordo com uma tese apresentada por Crawford, a filosofia da responsabilidade individual pela saúde ajudou a construir o senso comum individualista e antagônico às políticas de bem-estar social, que é a essência do neoliberalismo.

Essa concepção de Psicologia da saúde, que incorpora na década de 90 alguns preceitos do modelo biopsicossocial, de Engel, é difundida internacionalmente, criando uma tradição de pesquisa quantitativa e comportamental que se torna hegemônica.

A Psicologia da saúde tradicional, interessada em investigar características individuais invariáveis e generalizáveis sem preocupação com o contexto, será questionada, no âmbito metodológico, a partir de um intenso debate que surge na década de 90 sobre pesquisa qualitativa em Psicologia (Santiago-Delfosse \& Chamberlain, 2008).
A Psicologia tradicional se desenvolveu dividida em duas grandes tendências, no que diz respeito à metodologia: uma corrente experimental, que manipula as condições de pesquisa e na qual os dados são analisados mediante diversos tratamentos estatísticos das variáveis dependentes, e outra corrente, a das diferenças individuais, que não interfere no campo de estudo, mas que também utiliza uma análise estatística, buscando padrões de covariação ou de dependência entre variáveis. O estudo das variáveis configura o que Toomela (2008) denominou Psicologia quantitativa. Joel Michell (2003), por sua vez, afirma que a Psicologia tradicional endossa o que ele denominou imperativo quantitativo, que pode ser definido como a visão que afirma somente ser possível estudar algo cientificamente se houver uma quantificação. Sua motivação é a ideia de que todos os atributos são fundamentalmente quantitativos, uma ideia originada dos pitagóricos, mas que, através da Teologia e da Filosofia, repercutiu na cultura ocidental contemporânea. A Revolução Científica, ocorrida no século XVII, caracterizou-se pela matematicidade da Física, que serviu de modelo para a quantificação da ciência como um todo (Michell, 2003).

A origem da quantificação na Psicologia encontra-se nos trabalhos de Gustav Fechner. Seus experimentos, contudo, estavam restritos ao desenvolvimento de um método de mensuração de eventos mentais privados, no contexto da discussão metafísica sobre a relação mente-corpo. De acordo com Danziger (1990), autor que fundamenta toda a explanação deste parágrafo, a mensuração psicológica somente se tornou um instrumento usado em larga escala quando passou a ser interpretada como medida de uma capacidade individual, e não mais de uma experiência individual. Os fundamentos dessa estratégia de pesquisa que abordava a mensuração de capacidades psicológicas aparecem no trabalho de Hermann 
Ebbinghaus sobre memória (1913). O autor que mais contribuiu para a hegemonia, contudo, foi Edward Thorndike (1910), que construiu uma Psicologia quantitativa a partir do contexto da aprendizagem escolar. Para Thorndike, e para toda uma geração de psicólogos pesquisadores influenciados por ele, a aprendizagem não era uma atividade característica da criança escolar, mas uma categoria biológica fundamental aplicável a todos os seres vivos: "o que existe, existe em alguma quantidade", frase que resume bem a metafísica da quantificação do psicólogo estadunidense (Danziger, 1990). Através da Psicologia estadunidense, o projeto quantitativo se impôs internacionalmente.

Na Psicologia da saúde, o imperativo quantitativo se apresenta nos textos de Matarazzo, grande entusiasta do DSMIII, o Manual de Diagnóstico e Estatístico de Desordens Mentais da Associação de Psiquiatria Americana - Terceira Revisão, texto que representa a corrente quantitativa e biomédica na Medicina e na psiquiatria (Mayes \& Horwitz, 2005; Santiago-Delfosse \& Chamberlain, 2008). No editorial do primeiro número da Revista Health Psychology, revista oficial da 38 a Divisão da APA, George Stone (1982), ao comentar um artigo sobre os aspectos psicológicos da formação de políticas de saúde, afirma que uma das maiores necessidades dessa área é desenvolver medidas objetivas e quantitativas de valores de saúde que sejam aplicáveis a uma ampla variação de situações.

Durante os anos 70, surge no conjunto das ciências sociais uma corrente de pesquisa qualitativa que se interessa pela expressão do sujeito em situação, pela análise do discurso e dos contextos (Denzin \& Lincoln, 2006). As primeiras discussões na Psicologia sobre estratégias qualitativas de pesquisa são de Amendeo Giorgi (1970, 1978). Durante os anos 90, Schneider $(1998,1999)$ aponta a necessidade de uma corrente de pesquisa qualitativa na Psicologia, denominada por ele Psicologia romântica, que é constituída pelo desenvolvimento de um conjunto de abordagens qualitativas e subjetivas relativamente heterogêneas, a saber: Psicologia fenomenológica, Psicologia humanista, análise discursiva, etc. (Schneider, 1998). A abordagem qualitativa afirma que, em decorrência dos estudos estatísticos e de sua ênfase em generalização, a pesquisa quantitativa fez com que a Psicologia se esquecesse da singularidade do sujeito e do mundo no qual ele pensa e vive (Bruner, 1991; Schneider, 1998).

A Psicologia da saúde também serve como espaço para o debate metodológico, e vários autores questionaram a validade das pesquisas quantitativas no contexto da saúde. As pesquisas qualitativas no contexto da saúde deram uma atenção nova ao sujeito em situação, à psicologia da vida cotidiana concreta, e a linguagem passa a ser considerada um meio de acesso privilegiado à experiência humana (Santiago-Delfosse \& Chamberlain, 2008).

A Psicologia da saúde qualitativa surge, então, no final da década de 90 (Murray \& Chamberlain, 1998), e, aos poucos, alguns dos principais periódicos de Psicologia da saúde passam a publicar pesquisas qualitativas, destacando-se as revistas Journal of Health Psychology e Psychology and Health (Santiago-Delfosse \& Chamberlain, 2008).

Alguns autores, contudo, ainda no final da década de 90, começam a refletir acerca da crescente preocupação dos pesquisadores com a validação da pesquisa qualitativa, desenvolvendo uma certa metodolatria qualitativa, que contrastava com e que, paradoxalmente, complementava a metodolatria quantitativa dominante (Chamberlain, 2000). Esses autores afirmavam que a utilização dos métodos qualitativos, sem uma reflexão crítica acerca do seu 
objeto, pode conduzir a resultados mais pobres e reducionistas do que os obtidos pela abordagem quantitativa (SantiagoDelfosse \& Chamberlain, 2008). Os métodos qualitativos são ineficazes, por eles mesmos, para a resolução de questões internas da Psicologia da saúde (Chamberlain, 2000; Stam, 2000; Crossley, 2008). Como afirmam Santiago-Delfosse e Chamberlain, "para o progresso da disciplina, é necessário, além dos métodos, questionar os fundamentos epistemológicos e teóricos da Psicologia" (2008, p. 203).

Uma nova orientação surge, então, por parte de alguns autores, que, embora identificados com a abordagem qualitativa, defendiam a necessidade de uma crítica mais radical da Psicologia da saúde vigente; surge, então, a Psicologia da saúde crítica, marcada pela heterogeneidade de suas propostas.

Ian Parker (2007, p. 2) apresenta quatro elementos constituintes da Psicologia crítica:1) "um exame sistemático de como algumas variedades de experiências e ações psicológicas são privilegiadas em relação a outras, de como os discursos dominantes na Psicologia operam de modo ideológico e a serviço do poder", 2) o estudo das maneiras pelas quais todas as variedades de Psicologia são cultural e historicamente construídas, e como variedades de psicologias alternativas podem afirmar ou resistir a assunções ideológicas originadas nos modelos teóricos dominantes, 3) "estudo das formas de controle e autorregulação na vida cotidiana e dos meios pelos quais a cultura psicológica opera além das fronteiras da Academia e da prática profissional" (Parker, 2007, p. 3), 4) "investigação cotidiana das estruturas acadêmicas e do trabalho profissional da Psicologia e de como atividades cotidianas podem providenciar a base para a resistência a práticas disciplinares contemporâneas" (Parker, 2007, p. 3).
Fox, Sloan e Austin (2007) apresentam três tendências na Psicologia crítica desenvolvida na América do Norte: 1) concepção de novos métodos de pesquisa empírica em Psicologia e esforço para utilizá-los de modo respeitoso à condição humana com o propósito de reduzir as injustiças, favorecendo a mudança social progressiva ou radical, 2) análise e demonstração dos efeitos nefastos dos fundamentos ideológicos, empíricos e teóricos, caracterizados pelo positivismo e pelo individualismo, da Psicologia dominante, e o desenvolvimento, para substituí-la, de elementos teóricos e de práticas de pesquisa e de intervenção baseadas na complexidade da vida humana, respeitando o seu potencial de emancipação, 3) contestação do papel de autoproclamado especialista e o poder institucional de certa forma de Psicologia dominante sobre os indivíduos fragilizados, através de um processo de normalização da sociedade.

Santiago-Delfosse e Chamberlain (2008) descrevem dois grupos de psicólogos críticos; o primeiro, preocupado com a ciência psicológica, questiona os pressupostos epistemológicos, teóricos e metodológicos da Psicologia dominante; o segundo reivindica uma Psicologia engajada na sociedade, a fim de que as teorias, os métodos e as intervenções possam contribuir para a melhoria das condições de vida. Essas correntes se aproximaram, e, em 1999, no Canadá, realizaram a Primeira Conferência de Psicologia Crítica da Saúde, no período de 28 a 31 de julho (Murray, 2000). Dois anos depois, um grupo de pesquisadores de diversas nacionalidades, mas identificados predominantemente com a Psicologia comunitária, funda a International Society of Critical Health Psychology (Sociedade Internacional de Psicologia Crítica da Saúde).

De acordo com Santiago-Delfosse e Chamberlain, a Psicologia da saúde crítica apresenta as seguintes posições: 
questionamento da pertinência do modelo biopsicossocial para a compreensão da complexidade e da temporalidade da doença na experiência humana, refutação da ideologia a-contextualizada e individualista da corrente dominante da Psicologia da saúde e compreensão do papel e das práticas do psicólogo e questionamento de sua função e de sua formação. Apesar da divergência interna, essas posições caracterizam os pesquisadores identificados com a Psicologia da saúde crítica.

\section{Psicologia da saúde crítica e as abordagens ao campo da saúde}

De acordo com David Marks (2002), a Psicologia da saúde se desenvolveu com um significativo grau de especialização, com quatro abordagens que oferecem diferentes teorias, pesquisas e recomendações para a prática profissional. Há tensão entre elas em função de seus diferentes sistemas de valores. As abordagens são: Psicologia da saúde clínica, Psicologia da saúde pública, Psicologia da saúde comunitária e Psicologia da saúde crítica.

Psicologia da saúde clínica (PSCLIN): é a abordagem dominante, que é representada pela maioria dos manuais, dos periódicos científicos e dos programas acadêmicos. A PSCLIN reivindica uma fundamentação científica, é proclamada como a expressão de um modelo biopsicossocial de saúde, possui um vínculo estreito com a Psicologia clínica e é aplicada em clínicas, hospitais e centros de saúde.

Marks apresenta uma crítica ao modelo biopsicossocial a partir da constatação de que este efetivamente definiu o chamado modelo biopsicossocial, deixando aberto para que cada um o interpretasse como quisesse. Uma interpretação citada e criticada pelo psicólogo britânico é a da divisão trinta e oito (Psicologia da saúde da APA: "o produto de uma combinação de fatores incluindo características biológicas (por exemplo, predisposição genética), fatores comportamentais (estilo de vida, estresse e crenças em saúde) e condições sociais (influências culturais, relações familiares, suporte social)" (como citado em Marks, 2002, p. 10).

Para Marks, "o produto de uma combinação de fatores" não produz um modelo. Assim, o modelo biopsicossocial é uma peça de jargão técnico para descrever um conjunto de crenças sobre a saúde e a doença presentes na cultura e na Psicologia, que não descreve exatamente como processos psicossociais influenciam a saúde e a doença.

Psicologia da saúde pública (PSP): é um componente do sistema de saúde que lida mais com a promoção da saúde e a prevenção do que com o tratamento da doença. Dentro dessa abordagem, a Psicologia da saúde é vista como uma atividade multidisciplinar que busca integrar estudos epidemiológicos, intervenção e avaliação em saúde pública. Necessário se faz lembrar, contudo, que a promoção da saúde é uma atividade que envolve uma variedade de processos de defesa, negociação e construção comunitária. Marks (2002), citando um editorial publicado no British Journal of Health Psychology (Warde,2000), apresenta as seguintes oportunidades e desafios resultantes da convergência entre saúde pública e Psicologia da saúde: compreender e mudar comportamentos de saúde, meios de comunicação e informações sobre saúde, doença e risco e ensinar médicos como se comunicar efetivamente com seus pacientes e compreender porque alguns setores da sociedade são mais saudáveis do que outros. Uma Psicologia pública da saúde deve vincular-se obrigatoriamente a uma: 
Psicologia da saúde comunitária (PSCom): é baseada na pesquisa e na intervenção em comunidades, e envolve o trabalho em coalizão com membros de grupos e de comunidades vulneráveis e busca colaborar para o empoderamento desses indivíduos, visando a ajudá-los na superação das causas de suas vulnerabilidades (exclusão social e pobreza, por exemplo). Da mesma forma que a PSP, a PSCom compreende a saúde individual como o produto de determinantes políticos, econômicos e sociais, e conceitua saúde como Bem-Estar no sentido mais amplo do termo, o que inclui saúde física e mental como também resiliência. Uma perspectiva psico-comunitária sobre o desenvolvimento psicossocial saudável é, necessariamente, uma perspectiva ecológica. Aspectos como comunicação, cooperativismo e participação da sociedade civil também são temas pertinentes a essa abordagem.

Psicologia da saúde crítica (PSCri): objetiva analisar como o poder, os processos macrossociais e econômicos influenciam e/ ou estruturam a saúde, a atenção à saúde, a Psicologia da saúde e a sociedade como um todo. A PSCcri é preocupada com a natureza política de toda a existência humana, incorpora a Compaixão na teoria e na prática como elemento estruturante da abordagem, defende a liberdade de pensamento e é consciente da interdependência dos seres humanos como gentes sociais. Seu contexto de estudo é a sociedade como um todo, as políticas públicas e as relações de mercado. Essa abordagem faz uso de análise teórica, pensamento crítico, ação social e política, foca a promoção da saúde como principal estratégia, questiona conceitos como construção social da saúde, bemestar e qualidade de vida, afirmando o caráter problemático de tais expressões e desenvolvendo uma constante interrogação teórica acerca deles.

O psicólogo da saúde crítica utiliza um instrumental analítico crítico e evidências obtidas pelas outras três abordagens.

Marks propõe uma plataforma de integração das quatro abordagens mediante um modelo que organiza hierarquicamente a saúde em anéis concêntricos com o sistema de saúde se reorganizando de modo correspondente. Dessa forma, no anel central, encontram-se os fatores biológicos da saúde. O subsistema correspondente é o modelo biomédico de saúde; o recurso principal de intervenção da Psicologia da saúde é o aconselhamento. No primeiro anel, aparecem os fatores individuais relacionados ao estilo de vida. O subsistema de saúde relacionado contém a Medicina complementar, a nutrição, a fisioterapia e a Psicologia da saúde clínica, e utiliza também o aconselhamento como estratégia de intervenção; no segundo anel, as influências sociais e comunitárias predominam. $\mathrm{O}$ subsistema de saúde correspondente é formado pela epidemiologia, pela Sociologia médica, pela Medicina comunitária, pela saúde ambiental, pelo Serviço Social, pela Enfermagem comunitária e pelas organizações sociais. O terceiro anel é composto pelas condições de vida e trabalho, e o subsistema correspondente é formado por contribuições da saúde do trabalho, pela promoção da saúde, pela saúde pública e pela Psicologia da saúde pública. A Epidemiologia fundamenta os saberes desse nível. Por fim, no quarto anel, encontram-se as condições ambientais, econômicas, sociais, culturais e políticas. Os saberes do subsistema relacionado são: Economia, Antropologia médica, epidemiologia, política social e a Psicologia da saúde crítica.

A ética é o coração da atenção em saúde, e a comunicação é o seu modo de se envolver, mas ambos são fundamentos essenciais dos cinco subsistemas. A perspectiva crítica será necessária para uma reflexão à distância acerca dos dados obtidos em outros níveis (Marks, 2002). 
Algumas objeções foram feitas à proposta de Marks (2002) de apresentar a Psicologia da saúde crítica como uma das abordagens que compõem o campo teórico da Psicologia da saúde. Bolam e Chamberlain (2003) argumentam que a abordagem crítica fica em desvantagem se for compreendida como uma alternativa a outras formas de práticas em Psicologia da saúde, pois a orientação crítica pode ser aplicada a todos os tipos de prática (Prilleltensky \& Prilleltensky, 2003). Em consequência, de acordo com Bolam e Chamberlain, a crítica não deve ser vista como uma abordagem, mas como uma perspectiva que pode enformar uma prática reflexiva em todos os campos da Psicologia da saúde.

Saforcada (2006) oferece uma alternativa para a classificação da Psicologia crítica como abordagem. Sua análise dos sistemas de atenção à saúde se enquadra naquilo que foi por ele denominado Psicologia sanitária. O sistema total de saúde, objeto de estudo da Psicologia sanitária, é definido como

todo lo que una sociedad genera - de substancia material y/o ideacional, tangible y/o intangible - y pone en práctica en relación con los emergentes - positivos o negativos - del proceso de salud-enfermedad de quienes la constituyen y/o con el proceso mismo (Saforcada, 2006, p.43)

Assim, o campo da Psicologia da saúde é mais bem organizado da seguinte forma: perspectivas: tradicional e crítica; abordagens: Psicologia da saúde clínica, Psicologia da saúde pública, Psicologia da saúde comunitária e Psicologia sanitária.

\section{Psicologia da saúde crítica e prática profissional}

Prilleltensky e Prilleltensky (2003) oferecem uma proposta de prática da Psicologia da saúde a partir de uma perspectiva crítica. A proposta se fundamenta na promoção do bem-estar (individual, relacional e coletivo), na compreensão sobre o que é a boa vida, a boa sociedade, o conhecimento, a ética, o papel do profissional de saúde e o papel do usuário dos serviços de saúde. Deve haver um equilíbrio entre a necessária ênfase na autonomia dos usuários e a preocupação com o cuidado, com a compaixão e com a interdependência.

Os hospitais se mostram como um espaço de trabalho problemático em função do diferencial de poder existente nesses ambientes, o que torna mais difícil a tarefa de promover o empoderamento e a autodeterminação dos usuários. É preciso mostrar cuidado e compaixão pelos cidadãos que buscam os serviços de saúde, respeitando suas identidades sociais e encorajando-os a desenvolver suas habilidades para alcançarem metas pessoas, apesar de deficiência ou de doenças crônicas. É preciso permitir que as pessoas hospitalizadas possam apresentar seus pontos de vista acerca de sua saúde e respeitar a privacidade do paciente.

Nas comunidades, é preciso promover a colaboração e a participação democrática no desenvolvimento de ações de saúde. Necessário se faz envolver os membros da comunidade em atividades cívicas e de saúde. O psicólogo da saúde deverá criar parcerias com grupos da comunidade para promover justiça e cuidados com a saúde, agindo como um colaborador próximo, e não como um especialista distante. Necessário também se faz incentivar e promover os grupos de autoajuda como também considerar a possibilidade de alternativas ao tratamento médico, como dieta e mudança no estilo de vida.

Bolam e Chamberlain (2003), ao comentarem o artigo de Prilleltensky e Prilleltensky (2003), afirmam que uma prática em Psicologia da saúde a partir de uma perspectiva crítica 
envolve, necessariamente, uma discussão sobre o processo de profissionalização do campo. Os autores argumentam que o desenvolvimento da profissionalização em Psicologia da saúde seguiu o modelo da Psicologia clínica, que é derivado do modelo médico e predominantemente situado a serviço da biomedicina.

Especificamente, a adoção do modelo cientista-prático, que assume uma posição positivista na qual o psicólogo é compreendido como um experto que utiliza soluções técnicas, cientificamente comprovadas, para solucionar problemas sociais e individuais (Belar, 1997), sistematicamente ignora o contexto socio-histórico e os elementos políticos, morais e éticos da Psicologia da saúde. Como contraposição e essa perspectiva, Bolam e Chamberlain argumentam que uma prática crítica da Psicologia da saúde deve ser necessariamente uma prática reflexiva.

A reflexividade, de acordo com Bolam e Chamberlain (2003), pode ser entendida de várias maneiras, mas, de modo geral, envolve o reconhecimento do caráter contextualizado de todo conhecimento e de toda prática. Na Psicologia da saúde, a reflexividade deve ser um instrumento para transformar o modelo técnico de cientista-prático em um que defina o psicólogo da saúde como um agente social reflexivo e engajado. Entretanto, isso pode ocorrer em diferentes níveis. Os autores defendem uma reflexividade que questione a prática em um nível profundo, analisando os pressupostos que a fundamentam, questionando a que interesses esses pressupostos servem, questionando o conhecimento, como a prática enforma conhecimento e, mais amplamente, como conhecimento e práticas enformam a disciplina. É preciso, ainda, segundo Bolam e Chamberlain, distinguir reflexão no momento da prática (reflexão em ação) da reflexão posterior à prática (reflexão sobre a ação). A reflexão no momento da prática pode contribuir para uma prática mais tecnicamente qualificada, mas a reflexão sobre a prática necessariamente envolve a consideração de questões sobre poder, política e éticas que fundamentam a prática.

\section{Psicologia da saúde crítica e o contexto hospitalar}

Seild e Costa (1999), em um estudo sobre o trabalho do psicólogo na rede pública de Brasília, descreveram o modo como os psicólogos da saúde que atuavam em hospitais desenvolviam suas atividades. Havia dois modelos de atuação: o clínico e o de atenção integral à saúde. O modelo clínico era caracterizado por atendimentos individuais em um setor de serviço psicológico, com pouca ou nenhuma interação com as equipes de saúde. Em contraste, o modelo de atenção integral à saúde caracterizava-se por uma atuação difusa em diversos espaços do hospital, em interação constante com os demais profissionais da saúde, tendo como objetivo o atendimento de pacientes, familiares, equipe e comunidade. A pesquisa também indicou que os profissionais que atuavam de acordo com o modelo de atenção integral eram caracterizados por realização de pesquisa e maior interesse em contribuir para a construção de um corpo teórico prático da Psicologia da saúde. Infelizmente, nas pesquisas posteriores sobre a atuação do psicólogo no contexto hospitalar, o quadro descrito é bem diferente: predomínio do modelo clínico, falta de pesquisa, ênfase no atendimento individual (Yamamoto \& Cunha, 1998; Yamamoto, Trindade, \& Oliveira, 2002; Castro \& Bornholdt, 2004; Marcon, Luna, \& Lisboa, 2004; Sá, Lima, Santos, \& Clemente, 2005). O desafio que se apresenta, portanto, é o de oferecer um modelo de formação para o psicólogo da saúde no contexto hospitalar fundamentado em um modelo de atenção integral à saúde.

A Psicologia da saúde crítica é capaz de oferecer esse modelo. Algumas assunções 
dessa corrente são de grande utilidade para a formação do psicólogo em uma perspectiva de atenção integral à saúde:

Crítica ao modelo biopsicossocial de saúde: o modelo biopsicossocial de Engel, incorporado à Psicologia da saúde tradicional, é constituído por postulados psicodinâmicos bastante imprecisos e por uma tendência comportamental e objetivista. Alguns teóricos da Psicologia crítica recusamse a aceitar que o assim chamado modelo biopsicossocial seja de fato um modelo (Stam, 2000; Chamberlain, 2000; Crossley, 2001a; Crossley, 2001b, 2008; Marks, 2002). De fato, o modelo apenas reconhece a existência de três níveis de composição, mas não problematiza como eles se relacionam e também não apresenta um modelo de ser humano correspondente (Santiago-Delfosse \& Chamberlain, 2008).

Crossley é mais precisa em sua crítica à apropriação por parte da Psicologia da saúde tradicional do modelo biopsicossocial: um marco explicativo múltiplo, mas não integrado, no qual os fatores biológico, social e psicológicos coexistem de modo fragmentado. Além disso, por priorizar a abordagem quantitativa, o modelo biopsicossocial da Psicologia da saúde ignora a subjetividade, desconsidera a experiência humana do adoecer e produz conhecimentos simplistas visando a obter congruência com datas de investigações biomédicas, ou simplesmente adere a uma postura adaptadora através de instrumentos como a Escala de Ajuste Mental ao Câncer, desconsiderando a singularidade, a complexidade e a subjetividade da experiência humana.

Crossley, utilizando Habermas (1973) como referência, sugere que a Psicologia crítica da saúde se caracterize por transformar questões técnicas de cuidado e investigação sobre saúde e doença em questões morais e éticas, em um processo que deve ocorrer em dois níveis: primeiro, ouvir e relatar as vozes das pessoas ao descreverem suas experiências com saúde e doença de um modo ético e respeitoso; em seguida, identificar as estruturas de poder implícitas e explícitas que configuram as experiências dos usuários do sistema de cuidado com a saúde.

Prática profissional reflexiva: a literatura identifica quatro tipos de reflexividade que envolvem trabalhadores do campo da saúde: técnica, prática, processual e crítica (Yip, 2006). A reflexividade técnica relacionase ao conhecimento e à racionalidade técnica, e envolve habilidades para resolver problemas. A reflexividade prática busca respostas alternativas através de insights pessoais derivados e dirigidos à prática profissional. A reflexão crítica fundamentase em uma concepção emancipatória do conhecimento e visa a transformar a prática mediante mudanças nas condições sociais, políticas e culturais e nas forças estruturais que distorcem ou constrangem a prática profissional. Para Stam, a "reflexividade (...) significa reconhecer não somente a inevitabilidade de sempre ser situado, mas também decidir que bens morais nós iremos perseguir em nossas atividades" (2000, p. 279). A reflexividade crítica significa, então, reconhecer a dimensão moral do agir profissional.

Psicologia da saúde crítica e as microestruturas de poder: Prilleltensky e Prilleltensky(2003) ressaltam que a Psicologia da saúde crítica pode ser aplicada a contextos de microestruturas de poder. Especificamente, esses autores mencionam o contexto hospitalar e a relação médico-paciente, e acrescentam ainda que a advocacy, que pode ser traduzida como defesa/promoção de direitos, é uma prática fundamental para uma atuação transformadora no contexto hospitalar, e uma forma de institucionalizar essa prática é a ouvidoria hospitalar (Pereira, 
2002; Carvalho, Santana, \& Santana, 2009), que se configura como um espaço que vincula a questão do controle social dos serviços de saúde à temática da relação profissionalpaciente.

De acordo com Carvalho, Santana e Santana(2009), as ouvidorias hospitalares apresentam os seguintes desafios para a Psicologia da saúde: compreensão da micropolítica da instituição hospitalar, da instituição ouvidoria como instância micropolítica de controle social e produção de subsídios teóricos para capacitação de ouvidores, explicitando a dimensão política do trabalho na saúde. Uma Psicologia da saúde crítica certamente poderá enfrentar esses desafios.

\section{Considerações finais}

A Psicologia da saúde crítica é um movimento que se institucionaliza a partir do final da década de 90 do século passado, principalmente nos países de tradição anglo-saxônica. Surge como uma alternativa a uma Psicologia da saúde estruturada em torno de um suposto modelo biopsicossocial, inicialmente preocupado com o desenvolvimento de metodologias qualitativas de pesquisa. Perspectiva em franco desenvolvimento, a Psicologia da saúde crítica compreende o psicólogo como agente social e enfatiza a dimensão éticopolítica do trabalho em Psicologia da saúde, seja no âmbito da pesquisa, seja no âmbito da prática profissional.

No contexto hospitalar, a Psicologia da saúde crítica enfatiza a necessidade de, tanto no âmbito da pesquisa como no da intervenção, ouvir e relatar as vozes dos usuários dos serviços hospitalares a partir de uma perspectiva ética de respeito e de solidariedade. No âmbito político, necessário se faz identificar as estruturas de poder implícitas e explícitas das instituições hospitalares que enformam aas experiências dos pacientes. Um espaço estratégico para que essas práticas possam produzir efeitos transformadores e aperfeiçoem o controle social e a qualidade dos serviços é a ouvidoria, que precisa ser compreendida como um espaço estratégico de pesquisa e de intervenção pelos psicólogos da saúde críticos.

Para que isso possa ocorrer, no entanto, é preciso desenvolver uma reflexividade crítica sobre a prática, habilidade que precisa ser estimulada nos programas de formação em Psicologia, seja no nível da graduação, seja no nível da pós-graduação ou em qualquer espaço em que se desenvolva uma formação continuada em Psicologia da saúde. 


\section{Denis Barros de Carvalho}

Doutor em Psicologia Social pela Universidade Federal do Rio Grande do Norte e docente da Universidade Federal do Piauí, Teresina - PI - Brasil.

E-mail: denispsi@bol.com.br

\section{Endereço para envio de correspondência:}

Rua Trevos 1125, Morada do Sol. CEP: 64056-210. Teresina, PI.

\section{Referências}

Recebido 28/03/2011, 1a Reformulação 02/01/2013, Aprovado 22/01/2013.

Arnett, J. (2006). Psychology and health. Canadian Psychology, 47(1)19-32.

Belar, C. (1997). Clinical health psychology: A specialty for the $21^{\text {st }}$ century. Health Psychology, 16(5), 411-416.

Bernal, I., Sosa, A., Alfonso, L., Gómez, L., \& García, I. (2009). Formación de recursos humanos en psicología de la salud a partir de 1959 en Cuba. Revista Cubana de Saúde Pública, 35(1). Recuperado em 16 de agosto de 2009, de http:// scielo.sld.cu/scielo.php?script =sci_arttext $\&$ pid $=$ S0864$34662009000100006 \& \operatorname{lng}=\mathrm{es} \& \mathrm{nrm}=$ iso.

Bolam, B., \& Chamberlain, K. (2003). Professionalization and reflexivity in critical health psychology practice. Journal of Health Psychology, 8(2), 215-218.

Bruner, J. (1991). The narrative construction of reality. Critical Inquiry, 18(1), 1-21.

Carvalho, D., Santana, J., \& Santana, V. (2009). Humanização e controle social: o psicólogo como ouvidor hospitalar. Psicologia: Ciência \& Profissão, 29(1), 172-183.

Castro, E., \& Bornholdt, E. (2004). Psicologia da saúde $x$ psicologia hospitalar: definições e possibilidades de inserção profissional. Psicologia: Ciência \& Profissão, 24(3), 48-57.

Chamberlain, K. (2000). Methodolatry and qualitative health. Journal of Health Psychology, 5(3), 285-296.

Chiattone, H. (2000). A significação da psicologia no contexto hospitalar. In V. A. Angerami-Camon (Org.). Psicologia da saúde - um novo significado para a prática clínica (pp.73-165). São Paulo: Pioneira.

Correia, E. (2006). Uma visão fenomenológica-existencial em psicologia da saúde?! Análise Psicológica, 24(3), 337-341.

Crawford, R. (2006). Health as a meaningful social practice.
Health, 10(4), 401-420.

Crossley, M. (2000). Rethinking health psychology. Buckingham: Open University Press.

Crossley, M. (2001a). Rethinking psychological approaches towards health promotion. Psychology and Health, 16(2),161177.

Crossley, M. (2001b). Do we need to rethink health psychology? Psychology, Health and Medicine 6(3), 243-255.

Crossley, M. (2008). Critical health psychology: Developing and refining the approach. Social and Personality Psychology Compass, 2(1), 21-33.

Danziger, K. (1990). Constructing the subject: Historical origins of psychological research. Cambridge: Cambridge University press.

Denzin, N., \& Lincoln, Y. (2006). A disciplina e a prática da pesquisa qualitativa. In N. Denzin \& Y. Lincoln (Orgs.). $O$ planejamento da pesquisa qualitativa: teorias e abordagens (pp.15-41). Porto Alegre: Artmed; Bookman.

Ebbinghaus,H.(1913).Memory:a contribution to Experimental Psychology. Ney York:Teachers College.

Engel, G. (1977). The need for a new medical model: A challenge for biomedicine. Science, 196(4286), 129-136.

Fox, D., Sloan, T., \& Austin, S. (2008). Histoire et tendances de la psychologie critique en Amérique du Nord. Psychologie Française, 53, 157-171.

Giorgi, A. (1970). Psychology as human science. New York: Harper \& Row.

Giorgi, A. (1978). Problems encountered in developing a 
phenomenological approach to research. In F. Fransella (Org.). Personal construct psychology 1977 (pp.7-23). New York: Academic Press.

Guedes, C. (2006). A supervisão de estágio em psicologia hospitalar no curso de graduação: relato de uma experiência. Psicologia: Ciência \& Profissão, 26(3), 516-523.

Habermas, J. (1973). Theory and practice. Boston: Beacon.

Humbke, K., Brown, D., Welder, A., Fillion, D., \& Arnett, J. (2004). A survey of hospital psychology in Canada. Canadian Psychology, 45(1), 31-41.

Kelman, S. (1980). Social organization and the meaning of health. The Journal of Medicine and Philosophy, 5(2), 133-144.

Kenkel, M. B., DeLeon, P. H., Mantell, E. O., \& Steep, A. E. (2005). Divided no more: Psychology's role in integrated health care. Canadian Psychology, 46(3), 189-202.

Maia, E., Silva, N., Martins, R., \& Sebastiani, R. (2005). Psicologia da saúde-hospitalar: da formação à realidade. Universitas Psychologica, 14(1), 49-54.

Marcon, C., Luna, I., \& Lisboa, M. (2004). O psicólogo nas instituições hospitalares: características e desafios. Psicologia: Ciência \& Profissão, 24(1), 28-35.

Marks, D. (2002). Freedom, responsibility and power: Contrasting approaches to health psychology. Journal of Health Psychology, 7(1), 5-19.

Matarazzo, J. (1980). Behavioral health and behavioral medicine: Frontiers for a new health psychology. American Psychologist, 35(9), 807-817.

Matarazzo, J. (1982). Behavioral health's challenge to academic, scientific, and professional psychology. American Psychologist, 37(1), 1-14.

Matarazzo, J. D. (1984). Behavioral health: a 1990 challenge for health services professions. In J. D. Matarazzo, S. M. Weiss, J. A. Herd, N. E. Miller, \& S. M. Weiss (Eds.), Behavioral health (pp. 3-40). New York: John Wiley \& Sons.

Mayes, R. \& Horwitz, A. (2005). DSM-III and the revolution in the classification of mental illness. Journal of the History of the Behavioral Sciences, 41(3), 249-267.

Michell, J. (2003). The quantitative imperative: Positivism, naïve realism and the place qualitative methods in psychology. Theory \& Psychology, 13(1), 5-31.

Murray, M. (2000). Reconstructing health psychology : An introduction. Journal of Health Psychology, 5(3), 267-271.

Murray, M., \& Chamberlain, --. (1998). Qualitative Research in Health Psychology: Developments and Directions. Journal of Health Psychology, 3(3), 291-295.

Parker, I. (2007). Critical psychology: What it is and what it is not. Social and Personality Psychology Compass, 1(1), 1-15.

Pereira, L.(2002) . A voz do usuário no sistema hospitalar: ouvidorias. Sociologias UFRGS), Porto Alegre, 1(1), 82-120.

Prilleltensky, I., \& Prilleltensky, O. (2003). Towards a critical health psychology practice. Journal of Health Psychology, 8(2)197-210.

Romanov, R., \& Marchildon, G. (2003). Psychological services and the future of health care in Canada. Canadian Psychology, 44(4), 283-298.

Roso,A.(2007). Psicologia Social da Saúde: tornamo-nos eternamente responsáveis por aqueles que cativamos. Aletheia, 26,80-94.
Sá, A. Lima, A., Santos, I., \& Clemente, L. (2005). Psicólogo hospitalar da cidade de Recife-PE: formação e atuação. Psicologia: Ciência \& Profissão, 25(3), 384-397.

Safarcada,E. (2006). Psicologia Sanitaria :Análisis Crítico de los Sistemas de Atención de la Salud. Buenos Aires :Paidós.

Santiago-Delfosse, M., \& Chamberlain, K. (2008). Évolution des idées en psychologie de la santé dans le monde anglosaxon. De la psychologie de la santé (health psychology) à la psychologie critique de la santé (critical health psychology). Psychologie française, 53,195-210.

Schneider, K. (1998). Toward a science of the heart. American Psychologist, 53(3), 277-289.

Schneider, K. (1999). The revival of the romantic means a revival of psychology. Journal of Humanistic Psychology, 39(3), 13-39.

Seidl, E., \& Costa, A. (1999). O psicólogo na rede pública de saúde do Distrito Federal. Psicologia: Teoria e Pesquisa, 15(1), 27-35.

Stam, H. (2000). Theorizing health and illness: Functionalism, subjectivity and reflexivity. Journal of Health Psychology, 5(3), 273-283.

Stone,G.(1982).Health Psychology, a New Journal for a New Field. Health Psychology, 1(1)1-6.

Teixeira, J. (2002). Psicologia da saúde em Portugal: panorâmica breve. Análise Psicológica, 20(1), 651-170.

Teixeira, J. (2004). Psicologia da saúde. Análise Psicológica, 22(3), 441-448.

Teixeira. J. (2008). Psicologia da saúde crítica: breve revisão e perspectiva existencialista. Análise Psicológica, 26(2), 335-345.

Teixeira, J., Cima, M., \& Santa Cruz, C. (1999). Psicologia da saúde em Portugal. Análise Psicológica, 17(3), 435-455.

Thorndike,E. (1910). The contribution of psychology to education. Journal of Educational Psychology, 1, 5-12.

Tonetto, A., \& Gomes, W. (2005). Prática psicológica em hospitais: demandas e intervenções. Psico, 36(3), 283-291.

Tonetto, A., \& Gomes, W. (2007a). A prática do psicólogo hospitalar em equipe multidisciplinar. Estudos de Psicologia, 24(1), 89-98.

Tonetto, A., \& Gomes, W. (2007b). Competências e habilidades necessárias à prática psicológica hospitalar. Arquivos Brasileiros de Psicologia, 59(1), 38-50.

Toomela, A. (2008). Variables in psychology: A critique of quantitative psychology. Integr. Psych. Behav., 42, 245-265.

Trindade, I., \& Teixeira, J. (2002). Psicologia em serviços de saúde: intervenção em centros de saúde e hospitais. Análise Psicológica, 20(1), 171-174.

Warde,J. (2000). Editorial - Public Health Psychology:Expanding the horizons of Health Psychology.British Journal of Health Psychology, 5, 329-336.

Westra, H. A., Eastwood, J., Bouffard, B., \& Gerritson, C. (2006). Psychologists pursuit of prescriptive authority: Does it meet the goals of Canadian health care reform? Canadian Psychology, $47(2), 77-95$.

Yamamoto, O., \& Cunha, I. (1998). O psicólogo em hospitais de Natal: uma caracterização preliminar. Psicologia: Reflexão \& Crítica, 11(2), 345-362.

Yamamoto, O., Trindade, L., \& Oliveira, I. (2002). O psicólogo em hospitais no Rio Grande do Norte. Psicologia USP, 13(1), 217-246.

Yip, K. (2006). Reflectivity in social work practice with clients with mental-health illness. International Social Work, 49(2), 245-255. 